\title{
THEORETIC PERFORMANCE ANALYSIS OF A WATERMARKING SYSTEM BASED ON BERNOULLI CHAOTIC SEQUENCES
}

\author{
S. Tsekeridou, V. Solachidis, N. Nikolaidis, A. Nikolaidis, A. Tefas and \\ I. Pitas * \\ Department of Informatics, Aristotle University of Thessaloniki, \\ Box 451, Thessaloniki 54006, Greece \\ pitas@zeus.csd.auth.gr
}

\begin{abstract}
The paper statistically analyzes the behaviour of a simple blind copyright protection watermarking system based on chaotic watermark signals generated by $n$-way Bernoulli shift maps. The analysis involves theoretic evaluation of the system detection reliability, when a correlator detector is used. The effect of simple distortions on the detection reliability is also theoretically investigated. The aim of the paper is twofold: (i) to introduce the $n$-way Bernoulli shift generated chaotic watermarks and theoretically contemplate their properties with respect to detection reliability and (ii) to theoretically establish their potential superiority against the widely used pseudorandom watermarks.
\end{abstract}

Keywords: Theoretic analysis, watermarking, Bernoulli chaotic sequence

\section{INTRODUCTION}

The risk of illegal copying, reproduction and distribution of copyrighted material is becoming more threatening with the all-digital evolving solutions adopted by content providers, system designers and users, thus creating a pressing demand for copyright protection of multimedia content. This demand has been lately addressed by the emergence of a variety of watermarking methods. For an overview, the reader may look in [1]. The main trend is to use pseudorandom watermarks, which attain important properties for a watermarking application, such as autocorrelation function in the form of a Dirac delta function, unpredictability and statistical undetectability. Furthermore, the most widely used detector

*This work has been supported by the European Project IST-1999-10987 CERTIMARK

The original version of this chapter was revised: The copyright line was incorrect. This has been corrected. The Erratum to this chapter is available at DOI: 10.1007/978-0-387-35413-2_36 
is the correlator. Up to now, the foundation for performance evaluation of the majority of watermarking methods has been mostly experimental usually without any theoretical justification of their efficiency. Only few studies on a theoretical basis have been made $[2,3]$.

This paper statistically analyzes the behaviour of chaotic watermark signals generated by $n$-way Bernoulli shift maps. For this purpose, a simple blind copyright protection watermarking system is considered. Chaotic watermarks attain similar desirable properties with pseudorandom ones with the additional feature of controllable spectral/correlation properties, a fact that renders them ideal for a variety of applications.

\section{WATERMARKING SYSTEM MODEL}

Let $X_{i}, i \in[1, N]$ be samples of the 1-D continuous valued host signal $\mathrm{X}$ of length $N$. Let also $V_{i}, i \in[1, N]$ be the samples of the continuous valued watermark signal $\mathbf{V}$ generated by a watermark generation function $g: \mathbf{V}=g(N, K) . K$ denotes the watermark key. The watermark signal is assumed to be zero mean i.e. $E\left[V_{i}\right]=0$. We assume that $\mathbf{V}$ is embedded additively to $\mathbf{X}$, thus generating the watermarked signal $\mathbf{X}_{w}=\mathbf{X}+p \mathbf{V}$, where $p$ determines the watermark strength.

Given a signal $\mathbf{S}^{\prime}$, watermark detection aims at finding whether $\mathbf{S}^{\prime}$ hosts a certain watermark $\mathbf{W}^{\prime}=g\left(N, K^{\prime}\right)$. Thus, watermark detection can be formulated as a hypothesis test with the two hypotheses being:

$H_{0}: \mathbf{W}^{\prime}$ is indeed embedded in $\mathbf{S}^{\prime}$

$H_{1}: \mathbf{S}^{\prime}$ does not host $\mathbf{W}^{\prime}$

Event $H_{1}$ occurs either if $\mathbf{S}^{\prime}$ is not watermarked (event $H_{1 a}$ ) or if $\mathbf{S}^{\prime}$ is marked with a different watermark $\mathbf{W}=g\left(N, K^{\prime \prime}\right)$ than the one that we are trying to detect (event $H_{1 b}$ ). Thus $H_{1}=H_{1 b} \cup H_{1 a}$ where $H_{1 a}$, $H_{1 b}$ are mutually exclusive. If the host signal has not been distorted, the form of $\mathbf{S}^{\prime}$ for the three events $H_{0}, H_{1 a}, H_{1 b}$ can be summarized in: $\mathbf{S}^{\prime}=\mathbf{S}+p \mathbf{W}$. For $p=0$, this results in $\mathbf{S}^{\prime}=\mathbf{S}$ which corresponds to $H_{1 a}$, whereas for $p \neq 0$ and $\mathbf{W}=\mathbf{W}^{\prime}, \mathbf{S}^{\prime}=\mathbf{S}+p \mathbf{W}^{\prime}$ which corresponds to $H_{0}$. Finally, for $p \neq 0$ and $\mathbf{W} \neq \mathbf{W}^{\prime}$, we get the form of $\mathbf{S}^{\prime}$ under $H_{1 b}$.

During detection, the correlation $c$ between $\mathbf{S}^{\prime}$ and $\mathbf{W}^{\prime}$ is calculated: $c=\frac{1}{N} \sum_{i=1}^{N}\left(S_{i} W_{i}^{\prime}+p W_{i} W_{i}^{\prime}\right)$. In order to decide on the valid hypothesis, $c$ is compared against a suitably selected threshold $T$. The system performance for a given threshold can be measured in terms of the false alarm probability $P_{f a}(T)=\operatorname{Prob}\left\{c>T \mid H_{1}\right\}=\int_{T}^{\infty} f_{H_{1}}(t) d t$, (i.e., the probability to detect a watermark in a signal that is not watermarked or is watermarked with a different watermark) and the probability of false rejection $P_{f r}(T)=\operatorname{Prob}\left\{c<T \mid H_{0}\right\}=\int_{-\infty}^{T} f_{H_{0}}(t) d t$ (i.e., the probability to erroneously neglect the watermark existence in the signal). $f_{H_{0}}, f_{H_{1}}$ 
are the pdfs of $c$ under hypotheses $H_{0}, H_{1}$ respectively. By solving these for the independent variable $T$ and equating the results, $P_{f r}$ is expressed as a function of $P_{f a}$. The plot of $P_{f a}$ versus $P_{f r}$ is called the receiver operating characteristic (ROC) curve of the watermarking system. This curve conveys all the necessary system performance information. For the studied watermark sequences, $f_{H_{0}}, f_{H_{1 a}}, f_{H_{1 b}}$ are normal distributions, as will be shown. Then, the ROC curve is given by:

$$
P_{f a}=\frac{1}{2}\left[1-\operatorname{erf}\left[\frac{\sqrt{2} \sigma_{H_{0}} \operatorname{erf}^{-1}\left(2 P_{f r}-1\right)+\mu_{H_{0}}-\mu_{H_{1}}}{\sqrt{2} \sigma_{H_{1}}}\right]\right]
$$

The mean value of $c, \mu_{c}$, and its variance, $\sigma_{c}^{2}$, for both $H_{0}$ and $H_{1}$, assuming independence between $\mathbf{W}$ and $\mathbf{S}$, are given by:

$$
\begin{aligned}
\mu_{c}= & \frac{1}{N} \sum_{i=1}^{N} E\left[S_{i}\right] E\left[W_{i}^{\prime}\right]+\frac{1}{N} \sum_{i=1}^{N} p E\left[W_{i} W_{i}^{\prime}\right] \\
\sigma_{c}^{2}= & \frac{1}{N^{2}}\left[\sum_{i=1}^{N}\left(E\left[S_{i}^{2}\right] E\left[{W^{\prime}}_{i}^{2}\right]+p^{2} E\left[{W_{i}^{\prime 2}}_{i}^{2} W_{i}^{2}\right]+2 p E\left[S_{i}\right] E\left[W_{i}{W_{i}^{\prime 2}}_{i}^{\prime}\right]\right)+\right. \\
& \sum_{i=1}^{N} \sum_{j=1, j \neq i}^{N}\left(E\left[S_{i} S_{j}\right] E\left[W_{i}^{\prime} W_{j}^{\prime}\right]+p E\left[S_{i}\right] E\left[W_{i}^{\prime} W_{j} W_{j}^{\prime}\right]+\right. \\
& \left.\left.p E\left[S_{j}\right] E\left[W_{i} W_{j}^{\prime} W_{i}^{\prime}\right]+p^{2} E\left[W_{i} W_{j} W_{i}^{\prime} W_{j}^{\prime}\right]\right)\right]-\mu_{c}^{2}
\end{aligned}
$$

Signal $\mathbf{S}$ is assumed wide-sense stationary, i.e., $\mu_{S}=E\left[S_{i}\right], E\left[S_{i} S_{i+k}\right]=$ $\Gamma_{S, S}(k), \forall i \quad i \in[1, N]$, described by a first order autocorrelation function $\Gamma_{S, S}(k)=\mu_{S}^{2}+\sigma_{S}^{2} \alpha^{k}, k \geq 0$, where $\sigma_{S}^{2}$ is the signal variance and $\alpha=0.9, \cdots, 0.99[2]$.

\section{WATERMARKS GENERATED BY $N$-WAY BERNOULLI SHIFT MAPS}

$n$-way Bernoulli shifts $B_{n}(r)$ are chaotic maps defined by $\mathcal{B}_{n}:[0,1] \rightarrow$ $[0,1], r^{\prime}=B_{n}(r)=n r(\bmod 1)$. This map belongs to the class of piecewise affine Markov maps. A watermark sequence is generated by the map's recursive application, i.e. $W_{i+1}=B_{n}\left(W_{i}\right)=n W_{i}(\bmod 1), i \in$ $[1, N]$. The sequence starting point $W_{1}$ (map's initial condition) is considered as the watermark key $K$. The uniform distribution is an invariant probability density for the $n$-way Bernoulli shift maps $[4,5]$. Watermark signals (Bernoulli chaotic watermarks), generated in this way, are widesense stationary. To attain zero mean, the next modification is made:

$$
\begin{gathered}
\mathcal{B}_{n}:[-0.5,0.5] \rightarrow[-0.5,0.5] \\
W_{i+1}=B_{n}\left(W_{i}\right)=n\left(W_{i}+\frac{1}{2}\right)(\bmod 1)-\frac{1}{2}
\end{gathered}
$$


Sample $W_{i+k}$ is derived from sample $W_{i}$ through [5]:

$$
W_{i+k}=n^{k}\left(W_{i}+0.5\right)(\bmod 1)-0.5 \quad k>0
$$

Thus, the output of an $n$-way Bernoulli shift map after $k$ iterations (denoted by $B_{n}^{k}$ ) is equal to that of a $n^{k}$-way Bernoulli shift map:

$$
W_{i+k}=B_{n}^{k}\left(W_{i}\right)=B_{n^{k}}\left(W_{i}\right) \quad k>0
$$

If the starting point $W_{1}$ of a sequence generated by $n$-way Bernoulli shift maps is an irrational number, the sequence exhibits a chaotic, nonperiodic behaviour [6, 7]. Thus, if one considers two Bernoulli chaotic watermarks $\mathbf{W}, \mathbf{W}^{\prime}$ generated by the iterative application of the same map $B_{n}$ on any two randomly-chosen distinct, irrational starting points (watermark keys), then one of the following three cases may occur:

Case 1: The two watermarks are parts of the same chaotic orbit. This happens, if the starting points belong to the same chaotic orbit themselves, leading to a degree of correlation between $\mathbf{W}$ and $\mathbf{W}^{\prime}$. Crosscorrelation expressions become autocorrelation ones.

Case 2: The two watermarks belong to different chaotic orbits. In this case, they may be assumed independent. However, samples $W_{i}$ of the same watermark $\mathbf{W}$ are correlated with each other, since they are produced by iterating the same map on the same initial condition, a invalid statement for samples belonging to the same pseudorandom sequence.

Case 3: The two watermarks belong to eventually the same chaotic orbit. This happens when the map is initialized with two distinct irrational numbers, not of the same chaotic orbit, but the map iteration on those eventually leads to the same chaotic orbit after a certain number of iterations. Then, partial correlation may be observed between different watermarks. There might be seldom cases of two different watermarks differing only in the first few samples.

If two different watermarks are derived by initiating the map with two randomly chosen irrational numbers, there is no apriori knowledge on which of the above three cases will be valid. Since there is no possibility to control the occurrence of Cases 2 and 3, by appropriately selecting initial points during the watermark generation process, while this possibility exists for Case 1 (we just have to initialize the map with two irrational numbers belonging to the same chaotic orbit), extensive system performance analysis will be pursued for this case only.

If one considers two Bernoulli chaotic watermarks $\mathbf{W}, \mathbf{W}^{\prime}$ generated by the iterative application of the same map $B_{n}$ on two distinct, irrational starting points $W_{1}, W_{1}^{\prime}$, of the same chaotic orbit, there will always be an integer $k>0$ such that $W_{1}^{\prime}=B_{n}^{k}\left(W_{1}\right)$ OR $W_{1}=B_{n}^{k}\left(W_{1}^{\prime}\right)$. Consequently, their samples $W_{i}^{\prime}, W_{i}$ will also be associated: $W_{i}^{\prime}=W_{i+k}=$ 


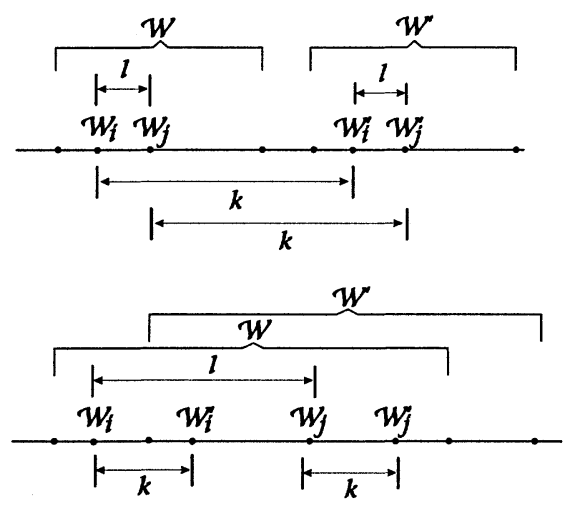

Figure 1 Watermark $\mathbf{W}^{\prime}=B_{n^{k}}(\mathrm{~W})$, assumed to be shifted by $k$ with respect to $\mathbf{W}$, may not overlap with $\mathbf{W}$ if $k \geq N$ (top), or may overlap with it if $k<N$ (bottom). The distance between two watermark samples, indexed by $i$ and $j$, is $l$.

$B_{n}^{k}\left(W_{i}\right)$ OR $W_{i}=W_{i+k}^{\prime}=B_{n}^{k}\left(W_{i}^{\prime}\right), \forall i, i=1 \ldots N$. These corollaries are used for the derivation of the joint moments of Bernoulli chaotic watermarks. A schematic representation of the above expressions can be seen in Figure 1. By exploiting the fact that the uniform distribution is an invariant density of the Bernoulli maps, one can thus calculate the $m$-order moments for $W_{i}$ :

$$
E\left[W_{i}^{m}\right]=\int_{-0.5}^{0.5} x^{m} f(x) d x=\left\{\begin{array}{cll}
0 & m \text { odd } \\
\frac{1}{(m+1) 2^{m}} & m \text { even }
\end{array}\right.
$$

One of the joint moments is the autocorrelation function (acf) $R_{W W}(k)$ :

$$
R_{W W}(k)=E\left[W_{i} W_{i+k}\right]=\frac{1}{12 n^{k}} \quad k \geq 0
$$

The other joint moments of $\mathbf{W}, \mathbf{W}^{\prime}$, for Bernoulli chaotic watermarks, appearing in (2), (3), can be derived in a straightforward manner. By observing (8), one concludes that $W_{i}$ and $W_{i+k}$ are correlated for small values of $k$ and $n$. Convergence, though, occurs quickly as $k$ increases, even for small $n$. As $n$ increases, the acf approximates a Dirac delta function, i.e., the acf, ideally, of random watermarks. Thus, $n$ controls the correlation properties of Bernoulli chaotic watermarks. Given $R_{W W}(k)$, their power spectral density (psd) $S_{W}(\omega)$ is evaluated as the Fourier transform of $R_{W W}(k)$ :

$$
S_{W}(\omega)=\sum_{k=-\infty}^{\infty} R_{W W}(k) e^{-j \omega k}=R_{W W}(0)+\sum_{k=1}^{\infty} R(k)\left(e^{j \omega k}+e^{-j \omega k}\right)
$$




$$
=\frac{1}{12} \frac{n^{2}-1}{n^{2}-2 n \cos (\omega)+1}
$$

where $-\pi \leq \omega \leq \pi$. It is easily observed that, for small values of $n$, Bernoulli chaotic watermarks are characterized by lowpass spectrums, while, as $n$ increases, the latter tend to be white, thus converging towards the spectrum of random watermarks. In short, $n$ proves to control the correlation/spectral properties of Bernoulli chaotic watermarks. By appropriately choosing $n$, Bernoulli chaotic watermarks can attain the best possible performance for the application at hand.

Substituting the evaluated expressions in (2) and (3), analytical expressions for $\mu_{c}$ and $\sigma_{c}^{2}$ are derived:

$$
\begin{gathered}
\mu_{c}=\frac{p}{12 n^{k}} \\
\sigma_{c}^{2}= \\
\left\{\begin{array}{l}
\frac{1}{12 N}\left[\sigma_{S}^{2}+\mu_{S}^{2}+p^{2}\left(\frac{1}{12}-\frac{1}{60 n^{2 k}}\right)\right]+\frac{p^{2} k}{144 N^{2}}\left(\frac{1}{n^{2}}\right)^{k}(2 N-(k+1))+ \\
\frac{\mu_{S}^{2}}{6 N^{2}(n-1)}\left[\left(N-k\left(\frac{1}{n}\right)^{k}-(N-k)\left(\frac{1}{n}\right)^{N-1}\right)-\right. \\
\left.\frac{n}{n-1}\left(1-k\left(\frac{1}{n}\right)^{k}+k\left(\frac{1}{n}\right)^{k+1}-(N-k)\left(\frac{1}{n}\right)^{N-1}+(N-k-1)\left(\frac{1}{n}\right)^{N}\right)\right]+ \\
\frac{\alpha \sigma_{S}^{2}}{6 N^{2}(n-\alpha)}\left[\left(N-k\left(\frac{\alpha}{n}\right)^{k}-(N-k)\left(\frac{\alpha}{n}\right)^{N-1}\right)-\right. \\
\left.\frac{n}{n-\alpha}\left(1-k\left(\frac{\alpha}{n}\right)^{k}+k\left(\frac{\alpha}{n}\right)^{k+1}-(N-k)\left(\frac{\alpha}{n}\right)^{N-1}+(N-k-1)\left(\frac{\alpha}{n}\right)^{N}\right)\right]+ \\
\frac{p^{2}}{12 N^{2}\left(n^{2}-1\right)}\left(\frac{-1}{5 n^{2 k}}\right)\left[\left(N-k\left(\frac{1}{n^{2}}\right)^{k}-(N-k)\left(\frac{1}{n^{2}}\right)^{N-1}\right)-\right. \\
\left.\frac{n^{2}}{n^{2}-1}\left(1-k\left(\frac{1}{n^{2}}\right)^{k}+k\left(\frac{1}{n^{2}}\right)^{k+1}-(N-k)\left(\frac{1}{n^{2}}\right)^{N-1}+(N-k-1)\left(\frac{1}{n^{2}}\right)^{N}\right)\right]+ \\
\frac{p^{2}}{7 N^{2}\left(n^{2}-1\right)}\left[\left(N\left(1+\left(\frac{1}{n^{2}}\right)^{k}\right)-2 k\left(\frac{1}{n^{2}}\right)^{k}-2(N-k)\left(\frac{1}{n^{2}}\right)^{N-1}\right)-\right. \\
\frac{n^{2}(1-1)}{n^{2}-1}\left(1-(k-1)\left(\frac{1}{n^{2}}\right)^{k}+k\left(\frac{1}{n^{2}}\right)^{k+1}-2(N-k)\left(\frac{1}{n^{2}}\right)^{N-1}+\right. \\
\left.\left.2(N-k-1)\left(\frac{1}{n^{2}}\right)^{N}\right)\right], \\
\frac{1}{12 N}\left[\sigma_{S}^{2}+\mu_{S}^{2}+p^{2}\left(\frac{1}{12}+\frac{N}{12 n^{2 k}}-\frac{1}{\left.\left.10 n^{2 k}\right)\right]+}\right.\right. \\
\frac{\mu_{S}^{2}}{6 N^{2}(n-1)}\left[N\left(1-\left(\frac{1}{n}\right)^{N-1}\right)-\frac{n}{n-1}\left(1-N\left(\frac{1}{n}\right)^{N-1}+(N-1)\left(\frac{1}{n}\right)^{N}\right)\right]+ \\
\frac{\left.p^{2}\right)}{12 N^{2}\left(n^{2}-1\right)}\left(\frac{-1}{5 n^{2 k}}+\frac{1}{6}\right)\left[N\left(1-\left(\frac{1}{n^{2}}\right)^{N-1}\right)-\frac{n^{2}}{n^{2}-1}\left(1-N\left(\frac{1}{n^{2}}\right)^{N-1}+\right.\right. \\
\left.\left.(N-1)\left(\frac{1}{n^{2}}\right)^{N}\right)\right]+\frac{\alpha \sigma_{S}^{2}}{6 N^{2}(n-\alpha)}\left[N\left(1-\left(\frac{\alpha}{n}\right)^{N-1}\right)-\frac{n}{n-\alpha}\left(1-N\left(\frac{\alpha}{n}\right)^{N-1}+\right.\right. \\
k>N-1 \\
\left.\left.(N-1)\left(\frac{\alpha}{n}\right)^{N}\right)\right],
\end{array}\right.
\end{gathered}
$$

These expressions can be used to obtain $\mu_{c}$ and $\sigma_{c}^{2}$ for events $H_{0}(\mathbf{W}=$ $\left.\mathbf{W}^{\prime}\right)$ by setting $k=0, H_{1 b}\left(\mathbf{W} \neq \mathbf{W}^{\prime}\right)$ by setting $k \neq 0$ and $H_{1 a}$ by setting $p=0 . \sigma_{c}^{2}$ for event $H_{1 b}$ proves to be greater than that for event $H_{1 a} . \quad \mu_{c}$ is larger for small $k>0$ but converges to that for $H_{1 a}$, as $k$ increases. Thus, event $H_{1 b}$ is the worst case in terms of bigger probability errors than $H_{1 a}$ or $H_{1}$.

Although Bernoulli chaotic watermarks, generated as described in Case 1, prove to be correlated for small $k>0$, the Central Limit Theorem for random variables with small dependency [8] may be used to establish that $c$ attains a Gaussian distribution, even for event $H_{1 b}$ (assuming that $N$ is sufficiently large). Furthermore, under the worst case 
assumption, both $\mu_{c}$ and $\sigma_{c}^{2}$ converge to a constant value for large $k$. For such $k, R_{W W}(k)=0$ meaning that $E\left[W_{i} W_{j}\right]=E\left[W_{i}\right] E\left[W_{j}\right]=0$. Thus, the terms of the sum in the correlation expression can be considered sufficiently independent and the distribution of $c$ under event $H_{1 b}$ for $k \rightarrow \infty$ can be assumed normal. In such a case, $P_{f a, H_{1 b}}$ can be estimated using the limit values of $\mu_{c}$ and $\sigma_{c}^{2}$ as $k \rightarrow \infty$. This is done since convergence is actually quickly reached leading to a very small probability (for large $N$ ) of actually facing a case where $k$ is rather small. $P_{f r}$ values are estimated using the values of $\mu_{c}$ and $\sigma_{c}^{2}$ for $k=0$ and ROC curves are evaluated from (1).

\section{PSEUDORANDOM WATERMARKS}

Zero-mean pseudorandom sequences in $[-0.5,0.5]$ are considered. Such sequences attain a white spectrum. Furthermore, for such watermarks, the terms of the sum in $c$ can be safely assumed to be sufficiently independent. Thus, due to the Central Limit Theorem, $c$ attains a Gaussian distribution for a sufficiently large $N$. Based on their properties, the moments of (2), (3), are easily obtained: $E\left[W_{i}^{m}\right]$ are given by (7) and $E\left[W_{i}^{l} W_{j}^{m}\right]=E\left[W_{i}^{l}\right] E\left[W_{j}^{m}\right]$. Similar expressions can be derived for the moments involving more than two random variables. Using these, $\mu_{c}$ and $\sigma_{c}^{2}$ are calculated equal to:

$$
\mu_{c}=\left\{\begin{array}{cl}
\frac{p}{12} & \text { if } \mathbf{W}=\mathbf{W}^{\prime} \\
0 & \text { if } \mathbf{W} \neq \mathbf{W}^{\prime} \\
0 & \text { if } p=0
\end{array} \quad \sigma_{c}^{2}=\left\{\begin{array}{cl}
\frac{1}{12 N}\left(\mu_{S}^{2}+\sigma_{S}^{2}+\frac{p^{2}}{15}\right) & \text { if } \mathbf{W}=\mathbf{W}^{\prime} \\
\frac{1}{12 N}\left(\mu_{S}^{2}+\sigma_{S}^{2}+\frac{p^{2}}{12}\right) & \text { if } \mathbf{W} \neq \mathbf{W}^{\prime} \\
\frac{1}{12 N}\left(\mu_{S}^{2}+\sigma_{S}^{2}\right) & \text { if } p=0
\end{array}\right.\right.
$$

It is seen that $c$ attains the same mean value for both events $\mathbf{W} \neq \mathbf{W}^{\prime}$ and $p=0$, while its variance for the first event is larger than that for the second, proving that the first event is the worst case.

\section{NOISE ADDITION}

$\mathbf{S}^{\prime}=\mathbf{S}+p \mathbf{W}$ is assumed to be corrupted by additive random white i.i.d. noise $\epsilon$ uniformly distributed in the interval $\left[-\epsilon_{r}, \epsilon_{r}\right]$. $\epsilon$ has zero mean value, $\mu_{\epsilon}=0$, and variance equal to $\sigma_{\epsilon}^{2}=\epsilon_{r}^{2} / 3$. W may be either a chaotic or random watermark. Detection involves estimation of the correlation between the noise corrupted signal $\mathbf{S}^{\prime}+\epsilon$ and a watermark $\mathbf{W}^{\prime}$, i.e.: $c_{\epsilon}=\frac{1}{N} \sum_{i=1}^{N}\left(S_{i}^{\prime}+\epsilon_{i}\right) W_{i}^{\prime}$. $\epsilon$ does not modify the correlator's Gaussian distribution since it is independent of the other signals. In order to determine the influence of noise addition on the system detection reliability, the mean $\mu_{c_{\epsilon}}$ and variance $\sigma_{c_{\epsilon}}^{2}$ of $c_{\epsilon}$ are estimated. For Bernoulli watermarks, we get $\mu_{c_{\epsilon}}=\frac{p}{12 n^{k}}$, while for random ones $\mu_{c_{\varepsilon}}$ is 
given by (12). Its variance $\sigma_{c_{\epsilon}}^{2}$ is estimated by:

$$
\begin{aligned}
\sigma_{c_{\epsilon}}^{2}= & \frac{1}{N^{2}} E\left[\sum_{i=1}^{N}\left(\left(S_{i}+p W_{i}+\epsilon_{i}\right) W_{i}^{\prime}\right)^{2}+\right. \\
& \sum_{i=1}^{N} \sum_{j=1, j \neq i}^{N}\left(\left(S_{i}+p W_{i}+\epsilon_{i}\right) W_{i}^{\prime}\right)\left(\left(S_{j}+p W_{j}+\epsilon_{j}\right) W_{j}^{\prime}\right)-\mu_{c_{\epsilon}}^{2}=\sigma_{c}^{2}+\frac{\epsilon_{r}^{2}}{36}
\end{aligned}
$$

where $\sigma_{c}^{2}$ denotes the correlator variance under no distortions. It is seen that noise corruption is not a serious threat, since it does not affect the correlation mean and only slightly affects its variance, if its power is much lower than that of the original signal. Noise corruption affects the system performance similarly for either random or chaotic watermarks.

\section{LINEAR LOWPASS FILTERING}

We consider a moving average filter of length $2 F+1$ with impulse response $h_{i}=\frac{1}{2 F+1}, i \in[-F, F]$. The filtered signal $\mathbf{S}_{f}^{\prime}$ is obtained by the linear convolution: $S_{f, i}^{\prime}=S_{i}^{\prime} * h_{i}=\sum_{l=-F}^{F} h_{l} S_{i-l}^{\prime}, i \in[1, N]$. The correlator detector estimates now the correlation $c_{f}$ between $\mathbf{S}_{f}^{\prime}$ and a watermark $\mathbf{W}^{\prime}: c_{f}=\frac{1}{N} \sum_{i=1}^{N} \sum_{l=-F}^{F} h_{l}\left(S_{i-l}+p W_{i-l}\right) W_{i}^{\prime}$ Filtering does not also modify the correlator Gaussian distribution. $\mu_{c_{f}}$ is now given by $\mu_{c_{f}}=\frac{1}{N} \sum_{l=-F}^{F} h_{l} \sum_{i=1}^{N}\left(E\left[S_{i-l}\right] E\left[W_{i}^{\prime}\right]+p E\left[W_{i-l} W_{i}^{\prime}\right]\right)$. For the case of Bernoulli chaotic watermarks, this becomes:

$$
\mu_{c_{f}}=\left\{\begin{array}{c}
\frac{p}{12(2 F+1)} \frac{1}{n^{k}}\left[1+\frac{1-\left(\frac{1}{n}\right)^{F}-n+n^{F+1}}{n-1}\right], \quad k \geq F \\
\frac{p}{12(2 F+1)} \frac{1}{n^{k}}\left[1+\frac{1-\left(\frac{1}{n}\right)^{F}-n+n^{k+1}+n^{2 k}\left(1-\left(\frac{1}{n}\right)^{F-k}\right)}{n-1}\right], \\
0 \leq k<F
\end{array}\right.
$$

When random watermarks are used, we get:

$$
\mu_{c_{f}}= \begin{cases}\frac{p}{12(2 F+1)}, & \text { if } \mathbf{W}=\mathbf{W}^{\prime} \\ 0, & \text { if } p=0 \text { or } \mathbf{W} \neq \mathbf{W}^{\prime}\end{cases}
$$

Estimation of $\sigma_{c_{f}}^{2}$ is performed as follows:

$$
\begin{aligned}
\sigma_{c_{f}}^{2}= & \sum_{l=-F}^{F} h_{l}^{2} E\left[c^{2}\right]+ \\
& \frac{1}{N^{2}} \sum_{m=-F}^{F} \sum_{n=-F, n \neq m}^{F} h_{m} h_{n}\left[\sum_{i=1}^{N}\left(E\left[S_{i-m} S_{i-n}\right] E\left[W_{i}^{\prime 2}\right]+p^{2} E\left[W_{i-m} W_{i-n} W_{i}^{\prime 2}\right]\right)+\right. \\
& \left.\sum_{i=1}^{N} \sum_{j=1, j \neq i}^{N}\left(E\left[S_{i-m} S_{j-n}\right] E\left[W_{i}^{\prime} W_{j}^{\prime}\right]+p^{2} E\left[W_{i}^{\prime} W_{j}^{\prime} W_{i-m} W_{j-n}\right]\right)\right]-\mu_{c_{f}}^{2}
\end{aligned}
$$


In (16), $E\left[c^{2}\right]=\sigma_{c}^{2}+\mu_{c}^{2}$ denotes the 2nd moment of $c$ under no distortions for any kind of watermarks. For the case of Bernoulli chaotic watermarks, $\sigma_{c_{f}}^{2}$ has been numerically estimated for predefined values of $N, n$ and $F$. Such numerical estimation is prone to errors dependent on the computational accuracy. For random watermarks, an analytic form of $\sigma_{c_{f}}^{2}$ is easily derived.

\section{EXPERIMENTAL RESULTS}

The system is fed with two input signals: a music audio signal and a random uniformly-distributed signal, appropriately prefiltered to comply with the signal model. Slightly better performance is obtained in case of perfect validity of the model, i.e. random signal. Performance is measured for both Bernoulli and random watermarks to enable their comparison. The value of $p$ is set such that the watermarked signal has $\mathrm{SNR}=30 \mathrm{~dB} .10000$ keys are used in the experiments. ROC curve estimation is performed under the worst case assumption (event $H_{1 b}$ ).

Initially, experimentally evaluated statistical and spectral properties of the Bernoulli chaotic watermarks are compared with the analytical ones. The experimental curves are almost identical with the theoretical ones in the case of $R_{W W}(k)$, whereas they converge to the latter in the case of $S_{W}(\omega)$. Figure 2a illustrates the psd $S_{W}(\omega)$ of the Bernoulli chaotic watermarks for different values of $n(3,5,7,9,11)$, as well as that of random watermarks for comparison purposes. It is easily observed that the Bernoulli chaotic watermark spectrum is lowpass for small values of $n$ and it converges to that of random white watermarks as $n$ increases significantly. Their acf also converges to that of random white watermarks, i.e. to a Dirac delta function, with increasing $n$. Both observations justify our earlier remark about the controllable spectral/correlation properties of the Bernoulli chaotic watermarks.

The achieved ROC curves under no attack, for varying $n$ and the audio signal as input, are shown in Figure $2 \mathrm{~b}$. The coincidence of the theoretical and empirical ones is noted. Respective curves are illustrated for random watermarks. It is seen that the latter attain the best performance with respect to the overall error probability. However, as $n$ increases, the performance of Bernoulli watermarks is quickly converging to that of random ones, since their lowpass spectral properties tend to white with increasing $n$. Similar conclusions are drawn after noise addition. Noise of small power does not greatly affect the system performance: $P_{f a}$ and $P_{f r}$ values are only slightly increased.

The system performance is also evaluated after lowpass filtering. Figure 3a shows both the theoretically and experimentally derived ROC 


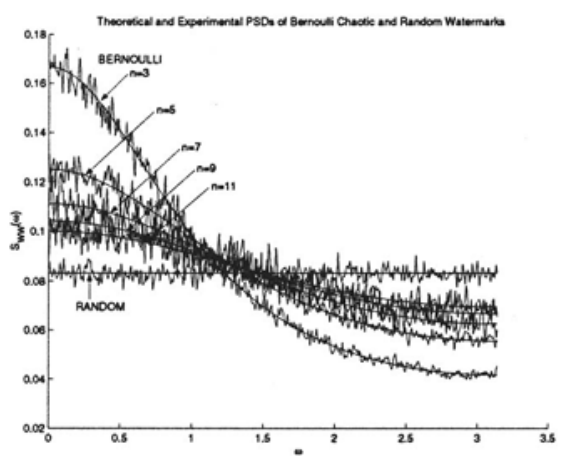

(a)

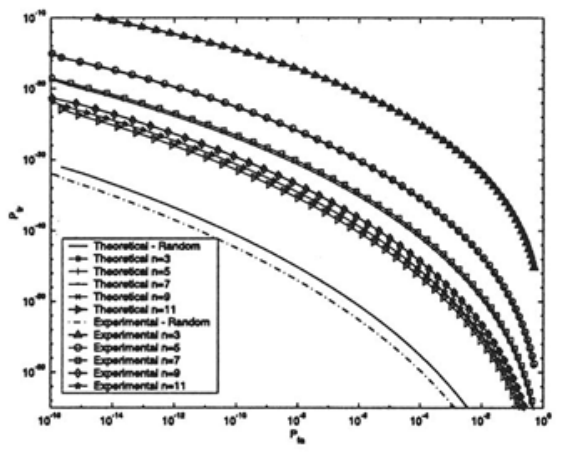

(b)

Figure 2 Theoretical and experimental evaluation of: (a) the psd of Bernoulli chaotic watermarks for different values of $n$, (b) ROCs for Bernoulli chaotic and random watermarks under no attack.

curves after moving average filtering (length 3 ) of the watermarked audio signal for Bernoulli and random watermarks. Both types of results tend to be similar. The numerical estimation of the theoretical $\sigma_{c f}^{2}$ for Bernoulli watermarks has its impact on the ROC curves. Use of the latter leads to better performance, especially for small values of $n$. This is easily justified by their lowpass spectral properties for small $n$, which renders them robust against lowpass distortions. To better establish this, lowpass filtering of random watermarks prior to embedding was done, so that they attain similar spectral/correlation properties with Bernoulli watermarks for various $n$. Figure $3 \mathrm{~b}$ shows the experimentally estimated ROC curves for LP random watermarks and for varying $n$, after moving average filtering of length 3 and for the random signal as input, compared against the ones obtained when using respective Bernoulli watermarks. They are nearly identical proving that the watermark spectrum plays a significant role in watermarking applications.

\section{CONCLUSIONS}

Bernoulli chaotic watermarks are introduced and their statistical properties relevant to watermarking are investigated to determine the overall system performance under no distortions or simple attacks. Similar investigation is performed for random watermarks, for comparison purposes. Bernoulli chaotic watermarks attain controllable spectral/correlation properties dependent on the value of $n$. They can be easily constructed with the appropriate spectral properties according to the prospective application or potential distortion, while preserving their invariant probability density. Random watermarks should be pre- 


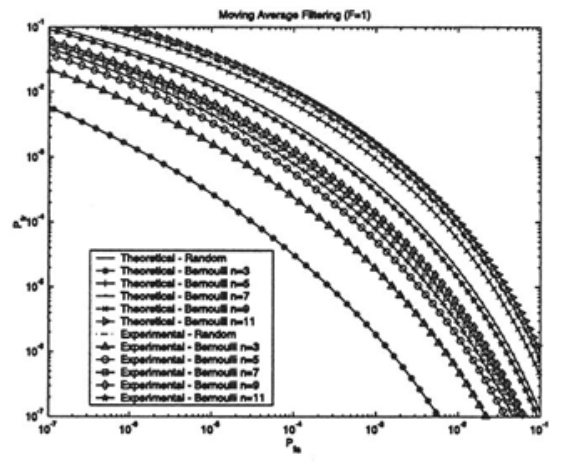

(a)

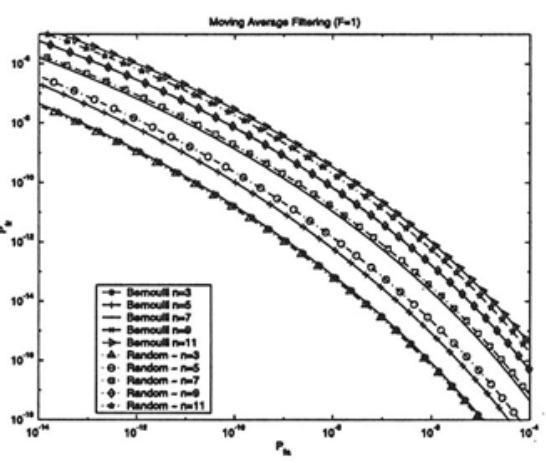

(b)

Figure 3 (a) Theoretical and empirical ROC curves for Bernoulli chaotic and random watermarks after lowpass filtering, and (b) empirical ROC curves for Bernoulli chaotic (solid lines) and random watermarks (dashed lines), prefiltered to attain similar spectral/correlation properties with the chaotic ones.

processed to exhibit similar efficiency. Such preprocessing modifies their initial distribution which enables the risk of possible perceptibility.

\section{References}

[1] "Special issue on identification \& protection of multimedia information", Proceedings of the IEEE, vol. 87, no. 7, 1999.

[2] G. Depovere, T. Kalker, and J.-P. Linnartz, "Improved watermark detection reliability using filtering before correlation", in Proc. of ICIP'98, 4-7 October 1998, vol. I, pp. 430-434.

[3] J.R. Hernandez and F. Perez-Gonzalez, "Statistical analysis of watermarking schemes for copyright protection of images", Proceedings of the IEEE, vol. 87, no. 7, pp. 1142-1166, July 1999.

[4] S.H. Isabelle and G.W. Wornell, "Statistical analysis and spectral estimation techniques for one-dimensional chaotic signals", IEEE Trans. on Signal Processing, vol. 45, no. 6, pp. 1495-1506, June 1997.

[5] R. Rovatti, G. Setti, and G. Mazzini, "Chaotic complex spreading sequences for asynchronous ds-cdma - part ii: Some theoretical performance bounds", IEEE Trans. on Circuits and Systems I, vol. 45, no. 4, pp. 496-506, April 1998.

[6] H.G. Schuster, Deterministic Chaos: An Introduction, Physik-Verlag, 1984.

[7] J. Froyland, Introduction to Chaos and Coherence, Institute of Physics, 1992.

[8] Patrick Billingsley, Probability and Measure, Wiley, 1995. 